\title{
The optical conductivity of graphene in the visible region of the spectrum
}

\author{
T. Stauber, ${ }^{1}$ N. M. R. Peres, ${ }^{1}$ and A. K. Geim ${ }^{2}$ \\ ${ }^{1}$ Centro de Física e Departamento de Física, Universidade do Minho, P-4710-057, Braga, Portugal and \\ ${ }^{2}$ Manchester Centre for Mesoscience and Nanotechnology, \\ University of Manchester, Manchester M12 9PL, United Kingdom
}

(Dated: October 22, 2018)

\begin{abstract}
We compute the optical conductivity of graphene beyond the usual Dirac cone approximation, giving results that are valid in the visible region of the conductivity spectrum. The effect of next nearest neighbor hoping is also discussed. Using the full expression for the optical conductivity, the transmission and reflection coefficients are given. We find that even in the optical regime the corrections to the Dirac cone approximation are surprisingly small (a few percent). Our results help in the interpretation of the experimental results reported by Nair et al. [Science 320, 1308 (2008)].

PACS numbers: 78.40.Ri,81.05.Uw,73.20.-r,78.66.Tr
\end{abstract}

\section{INTRODUCTION}

Graphene, an atomically thin material made only of carbon atoms arranged in a hexagonal lattice, was isolated only

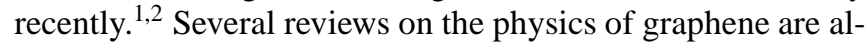
ready available in the literature , $^{3.4 .5 .6}$

At low energies, $E<1 \mathrm{eV}$, the electronic dispersion has the form $\epsilon(\boldsymbol{k})= \pm 3 t k a / 2$, where $t$ is the nearest neighbor hopping integral and $a$ is the carbon-carbon distance. The effective theory at these energy scales is that of a massless Dirac Hamiltonian in $(2+1)$ dimensions. If the experimental probes excite the system within this energy range, the Dirac Hamiltonian is all there is for describing the physics of graphene. On the other hand, for excitations out of this energy range it is necessary to include corrections to the Dirac Hamiltonian which will modify the energy spectrum and thus the density of states of the system. One immediate consequence is that the energy dispersion is no longer a function of the absolute value of the wave-number $k$. In this paper, we will calculate the optical conductivity of graphene including the leading corrections to the Dirac cone approximation.

One of the first calculations of the optical conductivity of graphene, using the Dirac Hamiltonian were done by Gusynin and Sharapov $\stackrel{\underline{7}}{\text { Th }}$ This first study was subsequently revisited a number of times $\frac{8,9,10}{9}$ and summarized in Ref. [11]. However, these authors did not include non-linear effects in the calculation. Also the effect of disorder was done on a phenomenological level, by broadening the delta functions into Lorentzians characterized by constant width $\Gamma$. We note that in the Dirac-cone approximation, the conductivity can also be obtained from the polarization. The calculations for finite chemical potential and arbitrary $|\boldsymbol{q}|$ and $\omega$ were done by Wunsch et al. .22 and Hwang and Das Sarma.$^{13}$

The calculation of the optical conductivity of graphene, in the Dirac Hamiltonian limit, including the effect of disorder in a self consistent way was done by Peres et al..$^{14}$ and recently also corrections due to electron-electron interaction were discussed. ${ }^{15,16}$ The calculation for the graphene bilayer with disorder was done by Koshino and Ando, 17 and by Nilsson et al. ${ }^{18}$ The optical conductivity of a clean bilayer was first computed by Abergel and Falko, $\frac{19}{19}$, and recently generalized to the biased ${ }^{20,21,22}$ bilayer case by Nicol and Carbotte. ${ }^{23}$
Within the Boltzmann approach, the optical conductivity of graphene was considered in Refs. [24,25], where the effect of phonons and the effect of mid-gap states were included. This approach, however, does not include transitions between the valence and the conduction band and is, therefore, restricted to finite doping. The voltage and the temperature dependence of the conductivity of graphene was considered by Vasko and Ryzhii, 26 using the Boltzmann approach. The same authors have recently computed the photoconductivity of graphene, including the effect of acoustic phonons ${ }^{27}$

The effect of temperature on the optical conductivity of clean graphene was considered by L. A. Falkovsky and A. A. Varlamovi ${ }^{28}$ The far-infrared properties of clean graphene were studied in Refs. 29 and 30. Also this study was restricted to the Dirac spectrum approximation.

It is interesting to note that the conductivity of clean graphene, at half filling and in the limit of zero temperature, is given by the universal value $\pi e^{2} /(2 h) \cdot 31,32$ On the other hand, if the temperature is kept finite the conductivity goes to zero at zero frequency, but the effect of optical phonons does not change the value of the conductivity of clean graphene ${ }^{33}$ This behavior should be compared with the calculation of the DC conductivity of disordered graphene, which for zero chemical potential presents the value of $4 e^{2} /(\pi h) ! 14,31,34,35$

From the experimental point of view, the work of Kuzmenko et al $\stackrel{36}{\underline{3}}$ studied the optical conductivity of graphite in the energy range $[0,1] \mathrm{eV}$, and showed that its behavior is close to that predicted for clean graphene in that energy range. An explanation of this odd fact was attempted within the Slonczewski-McClure-Weiss model. The complex dielectric constant of graphite was studied by Pedersen for all energy ranges ${ }^{37}$ The infrared spectroscopy of Landau levels in graphene was studied by Jiang et al $l^{\frac{38}{4}}$ and Deacon et al $l^{39}$, confirming the magnetic field dependence of the energy levels and deducing a band velocity for graphene of $1.1 \times 10^{6} \mathrm{~m} / \mathrm{s}$. Recently, the infrared conductivity of a single graphene sheet was obtained. ${ }^{40,41}$

Recent studies of graphene multilayers grown on $\mathrm{SiC}$ from $\mathrm{THz}$ to visible optics showed a rather complex behavior ${ }^{42}$ with values of optical conductivity close to those predicted for graphene at infrared frequencies as well as to those measured in graphite ${ }^{36}$. This experiment ${ }^{42}$ especially indicates the 
need for a graphene theory valid all the way to optical frequencies. The absorption spectrum of multilayer graphene in high magnetic fields was recently discussed in Ref. 43, including corrections to the Dirac cone approximation.

In this paper we address the question of how the conductivity of clean graphene changes when ones departs from the linear spectrum approach. This is an important question for experiments done in the visible region of the spectrum. ${ }^{44}$ The paper is organized as follows: in Sec. П we introduce our model and derive the current operator; in Sec. III] we discuss the optical conductivity of graphene by taking into account its full density of states; in Sec. IV we discuss the effect on the optical conductivity of a next nearest neighbors hopping term; in Sec. $\nabla$ we analyze the scattering of light by a graphene plane located at the interface of two different dielectrics and give the transmissivity and reflectivity curves in the visible region of the spectrum; finally in Sec. VI] we give our conclusions.

\section{THE HAMILTONIAN AND THE CURRENT OPERATORS}

The Hamiltonian, in tight binding form, for electrons in graphene is written as

$$
\begin{aligned}
H= & -t \sum_{\boldsymbol{R}, \sigma} \sum_{\boldsymbol{\delta}=\boldsymbol{\delta}_{1}-\boldsymbol{\delta}_{3}}\left[a_{\sigma}^{\dagger}(\boldsymbol{R}) b_{\sigma}(\boldsymbol{R}+\boldsymbol{\delta})+H . c .\right] \\
& -\frac{t^{\prime}}{2} \sum_{\boldsymbol{R}, \sigma} \sum_{\boldsymbol{\delta}=\delta_{4}-\boldsymbol{\delta}_{9}}\left[a_{\sigma}^{\dagger}(\boldsymbol{R}) a_{\sigma}(\boldsymbol{R}+\boldsymbol{\delta})+H . c .\right] \\
& -\frac{t^{\prime}}{2} \sum_{\boldsymbol{R}, \sigma} \sum_{\boldsymbol{\delta}=\delta_{4}-\boldsymbol{\delta}_{9}}\left[b_{\sigma}^{\dagger}(\boldsymbol{R}) b_{\sigma}(\boldsymbol{R}+\boldsymbol{\delta})+H . c .\right]
\end{aligned}
$$

where the operator $a_{\sigma}^{\dagger}(\boldsymbol{R})$ creates an electron in the carbon atoms of sub-lattice $A$, whereas $b_{\sigma}^{\dagger}(\boldsymbol{R})$ does the same in sublattice $B, t$ is the hopping parameter connecting first nearest neighbors, with a value of the order of $3 \mathrm{eV}$, and $t^{\prime}$ is the hopping parameter for second nearest neighbors, with a value of the order of $0.1 t$. The vectors $\delta_{i}$ are represented in Fig. 1 and have the form

$$
\begin{aligned}
& \boldsymbol{\delta}_{1}=\frac{a}{2}(1, \sqrt{3}), \boldsymbol{\delta}_{2}=\frac{a}{2}(1,-\sqrt{3}), \boldsymbol{\delta}_{3}=-a(1,0), \\
& \boldsymbol{\delta}_{4}=a(0, \sqrt{3}), \boldsymbol{\delta}_{5}=-\boldsymbol{\delta}_{4}, \boldsymbol{\delta}_{6}=\frac{3 a}{2}\left(1, \frac{1}{\sqrt{3}}\right) \\
& \boldsymbol{\delta}_{7}=-\boldsymbol{\delta}_{6}, \boldsymbol{\delta}_{8}=\frac{3 a}{2}\left(1,-\frac{1}{\sqrt{3}}\right), \boldsymbol{\delta}_{9}=-\boldsymbol{\delta}_{8}
\end{aligned}
$$

In order to obtain the current operator we modify the hopping parameters as

$$
t \rightarrow t e^{i \frac{e}{\hbar} \boldsymbol{A}(t) \cdot \boldsymbol{\delta}},
$$

and the same for $t^{\prime}$. Expanding the exponential up to second order in the vector potential $\boldsymbol{A}(t)$ and assuming that the electric field is oriented along the $x$ direction, the current operator

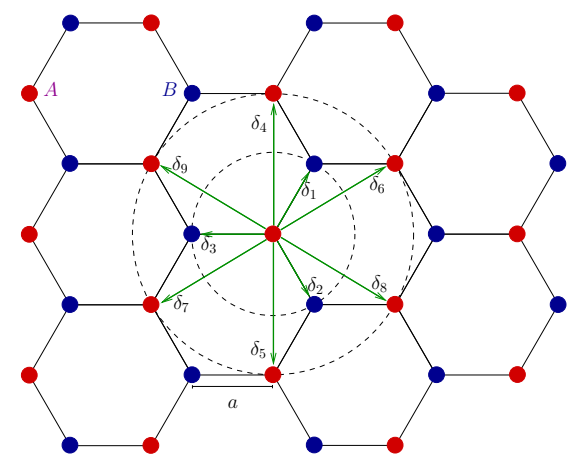

FIG. 1: (color online) Representation of the vectors $\boldsymbol{\delta}_{i}$, with $i=$ $1-9$. The carbon-carbon distance, $a$, and the $A$ and $B$ atoms are also depicted.

is obtained from

$$
j_{x}=-\frac{\partial H}{\partial A_{x}(t)},
$$

leading to $j_{x}=j_{x}^{P}+A_{x}(t) j_{x}^{D}$. The operator $j_{x}^{P}$ reads

$$
\begin{aligned}
j_{x}^{P} & =\frac{t i e}{\hbar} \sum_{\boldsymbol{R}, \sigma} \sum_{\boldsymbol{\delta}=\boldsymbol{\delta}_{1}-\boldsymbol{\delta}_{3}}\left[\delta_{x} a_{\sigma}^{\dagger}(\boldsymbol{R}) b_{\sigma}(\boldsymbol{R}+\boldsymbol{\delta})-H . c .\right] \\
& +\frac{t^{\prime} i e}{2 \hbar} \sum_{\boldsymbol{R}, \sigma} \sum_{\boldsymbol{\delta}=\boldsymbol{\delta}_{4}-\boldsymbol{\delta}_{9}}\left[\delta_{x} a_{\sigma}^{\dagger}(\boldsymbol{R}) a_{\sigma}(\boldsymbol{R}+\boldsymbol{\delta})-H . c .\right] \\
& +\frac{t^{\prime} i e}{2 \hbar} \sum_{\boldsymbol{R}, \sigma} \sum_{\boldsymbol{\delta}=\boldsymbol{\delta}_{4}-\boldsymbol{\delta}_{9}}\left[\delta_{x} b_{\sigma}^{\dagger}(\boldsymbol{R}) b_{\sigma}(\boldsymbol{R}+\boldsymbol{\delta})-H . c .\right]
\end{aligned}
$$

The operator $j_{x}^{D}$ can be found from the linear term in $A_{x}(t)$ expansion of the Hamiltonian.

\section{THE OPTICAL CONDUCTIVITY}

\section{A. The Kubo formula}

The Kubo formula for the conductivity is given by

$$
\sigma_{x x}(\omega)=\frac{<j_{x}^{D}>}{i A_{s}\left(\omega+i 0^{+}\right)}+\frac{\Lambda_{x x}\left(\omega+i 0^{+}\right)}{i \hbar A_{s}\left(\omega+i 0^{+}\right)},
$$

with $A_{s}=N_{c} A_{c}$ the area of the sample, and $A_{c}=3 \sqrt{3} a^{2} / 2$ ( $a$ is the carbon-carbon distance) the area of the unit cell, from which it follows that

$$
\Re \sigma_{x x}(\omega)=D \delta(\omega)+\frac{\Im \Lambda_{x x}\left(\omega+i 0^{+}\right)}{\hbar \omega A_{s}},
$$

and

$$
\Im \sigma_{x x}(\omega)=-\frac{<j_{x}^{D}>}{A_{s} \omega}-\frac{\Re \Lambda_{x x}\left(\omega+i 0^{+}\right)}{\hbar \omega A_{s}},
$$

where $D$ is the charge stiffness which reads

$$
D=-\pi \frac{<j_{x}^{D}>}{A_{s}}-\pi \frac{\Re \Lambda_{x x}\left(\omega+i 0^{+}\right)}{\hbar A_{s}} .
$$


The function $\Lambda_{x x}\left(\omega+i 0^{+}\right)$is obtained from the Matsubara current-current correlation function, defined as

$$
\Lambda_{x x}\left(i \omega_{n}\right)=\int_{0}^{\hbar \beta} d \tau e^{i \omega_{n} \tau}<T_{\tau} j_{x}^{P}(\tau) j_{x}^{P}(0)>.
$$

In what follows we start by neglecting the contribution of $t^{\prime}$ to the current operator. Its effect is analyzed later and shown to be negligible. The function $\Im \Lambda_{x x}\left(\omega+i 0^{+}\right)$is given by

$$
\begin{aligned}
& \Im \Lambda_{x x}\left(\omega+i 0^{+}\right)=\frac{t^{2} e^{2} a^{2}}{8 \hbar^{2}} \sum_{\boldsymbol{k}} f[\phi(\boldsymbol{k})] \\
\times & {\left[n_{F}(-t|\phi(\boldsymbol{k})|-\mu)-n_{F}(t|\phi(\boldsymbol{k})|-\mu)\right] } \\
\times & {[\pi \delta(\omega-2 t|\phi(\boldsymbol{k})| / \hbar)-\pi \delta(\omega+2 t|\phi(\boldsymbol{k})| / \hbar)], }
\end{aligned}
$$

where $n_{F}(x)$ is the usual Fermi distribution, $\mu$ is the chemical potential, and the function $\Re \Lambda_{x x}\left(\omega+i 0^{+}\right)$is given by

$$
\begin{aligned}
& \Re \Lambda_{x x}\left(\omega+i 0^{+}\right)=-\frac{t^{2} e^{2} a^{2}}{8 \hbar^{2}} \mathcal{P} \sum_{\boldsymbol{k}} f[\phi(\boldsymbol{k})] \\
\times & {\left[n_{F}(-t|\phi(\boldsymbol{k})|-\mu)-n_{F}(t|\phi(\boldsymbol{k})|-\mu)\right] } \\
\times & \frac{4 t|\phi(\boldsymbol{k})|}{\omega^{2}-(2|\phi(\boldsymbol{k})|)^{2}},
\end{aligned}
$$

with

$$
f[\phi(\boldsymbol{k})]=18-4|\phi(\boldsymbol{k})|^{2}+18 \frac{[\Re \phi(\boldsymbol{k})]^{2}-[\Im \phi(\boldsymbol{k})]^{2}}{|\phi(\boldsymbol{k})|^{2}},
$$

and $\mathcal{P}$ denoting the principal part of the integral. The graphene energy bands are given by $\epsilon(\boldsymbol{k})= \pm t|\phi(\boldsymbol{k})|$, with $\phi(\boldsymbol{k})$ defined as

$$
\phi(\boldsymbol{k})=1+e^{\boldsymbol{k} \cdot\left(\boldsymbol{\delta}_{1}-\boldsymbol{\delta}_{3}\right)}+e^{\boldsymbol{k} \cdot\left(\boldsymbol{\delta}_{2}-\boldsymbol{\delta}_{3}\right)} .
$$

\section{B. The real part of the conductivity}

The expression for (11) can almost be written in terms of the energy dispersion $\epsilon(\boldsymbol{k})$, except for the term

$$
\frac{[\Re \phi(\boldsymbol{k})]^{2}-[\Im \phi(\boldsymbol{k})]^{2}}{|\phi(\boldsymbol{k})|^{2}} .
$$

In order to proceed analytically, and for the time being (see Section [IIC), we approximate this term by its value calculated in the Dirac cone approximation (see appendix A)

$$
\frac{1}{N_{c}} \sum_{\boldsymbol{k}} \frac{[\Re \phi(\boldsymbol{k})]^{2}-[\Im \phi(\boldsymbol{k})]^{2}}{|\phi(\boldsymbol{k})|^{2}} g(|\phi(\boldsymbol{k})|) \simeq 0,
$$

where $g(|\phi(\boldsymbol{k})|)$ is some given function depending only on the modulus of $\phi(\boldsymbol{k})$. With this approximation, we have

$$
f[\phi(\boldsymbol{k})] \simeq 18-4|\phi(\boldsymbol{k})|^{2} .
$$

Introducing the density of states per spin per unit cell, $\rho(E)$, defined as

$$
\rho(E)=\frac{1}{N_{c}} \sum_{\boldsymbol{k}} \delta\left(E-t\left|\phi_{\boldsymbol{k}}\right|\right)
$$
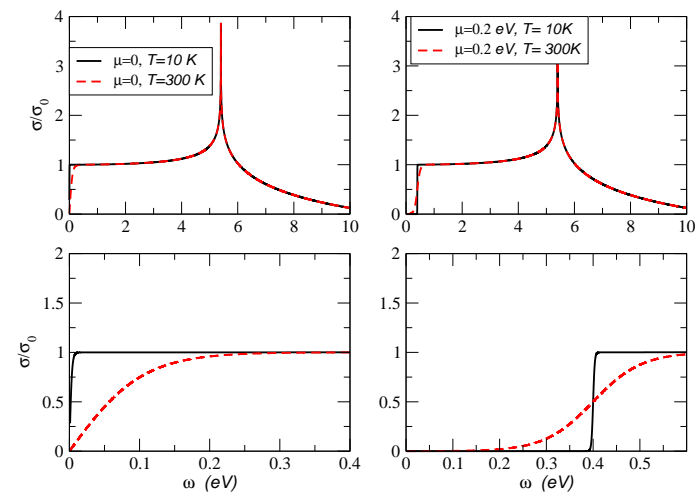

FIG. 2: (color online) The optical conductivity as function of frequency for two values of the chemical potential, $\mu=0 \mathrm{eV}$ and $\mu=0.2 \mathrm{eV}$, and two temperatures $T=10 \mathrm{~K}$ and $T=300 \mathrm{~K}$. The bottom panels are a zoom in, close to zero frequency, which allow to depict the frequency region where differences in the chemical potential and in temperature are most important. We have used $t=2.7 \mathrm{eV}$.

the expression for the real part of the conductivity reads

$$
\begin{aligned}
& \Re \sigma_{x x}(\omega)=\sigma_{0} \frac{\pi t^{2} a^{2}}{8 A_{c} \hbar \omega} \rho(\hbar \omega / 2)\left[18-(\hbar \omega)^{2} / t^{2}\right] \\
& \times\left[\tanh \frac{\hbar \omega+2 \mu}{4 k_{B} T}+\tanh \frac{\hbar \omega-2 \mu}{4 k_{B} T}\right] .
\end{aligned}
$$

Equation 19 is essentially exact in the visible range of the spectrum; missing is only the a contribution coming from Eq. 16. which contribution wil later be shown to be negligible. In the above equation $\sigma_{0}$ is

$$
\sigma_{0}=\frac{\pi}{2} \frac{e^{2}}{h}
$$

The momentum integral in Eq. (18) can be performed leading to

$$
\rho(E)=\frac{2 E}{t^{2} \pi^{2}} \begin{cases}\frac{1}{\sqrt{F(E / t)}} \mathbf{K}\left(\frac{4 E / t}{F(E / t)}\right), & 0<E<t, \\ \frac{1}{\sqrt{4 E / t}} \mathbf{K}\left(\frac{F(E / t)}{4 E / t}\right), & t<E<3 t,\end{cases}
$$

where $F(x)$ is given by

$$
F(x)=(1+x)^{2}-\frac{\left(x^{2}-1\right)^{2}}{4},
$$

and $\mathbf{K}(m)$ is defined as

$$
\mathbf{K}(m)=\int_{0}^{1} d x\left[\left(1-x^{2}\right)\left(1-m x^{2}\right)\right]^{-1 / 2} .
$$

In Figure 2 we give a plot of Eq. (19) over a large energy range, including the visible part of the spectrum $(E \in$ $[1.0,3.1] \mathrm{eV})$. 
It is useful to derive from Eq. (19) an asymptotic expansion for $\Re \sigma_{x x}(\omega)$. For that, we expand the density of states around $E=0$ and obtain

$$
\rho(E) \simeq \frac{2 E}{\sqrt{3} \pi t^{2}}+\frac{2 E^{3}}{3 \sqrt{3} \pi t^{4}}+\frac{10 E^{5}}{27 \sqrt{3} \pi t^{6}}
$$

Using Eq. (24) in Eq. (19) we obtain for the optical conductivity the approximate result

$$
\begin{aligned}
\Re \sigma_{x x}(\omega) & =\sigma_{0}\left(\frac{1}{2}+\frac{1}{72} \frac{(\hbar \omega)^{2}}{t^{2}}\right) \\
& \times\left(\tanh \frac{\hbar \omega+2 \mu}{4 k_{B} T}+\tanh \frac{\hbar \omega-2 \mu}{4 k_{B} T}\right)
\end{aligned}
$$

In the case of $\mu=0$ this expression is the same as in Kuzmenko et al..$^{36}$ and in Falkovsky and Pershoguba ${ }^{29}$ if in both cases the $(\hbar \omega / t)^{2}$ term is neglected.

\section{Correction to $\Re \sigma_{x x}(\omega)$ introduced by Eq. (16)}

We now want to make quantitative the effect of the term given by Eq. (16), which was neglected in Eq. (19). To that end we expand the function $\phi(\boldsymbol{k})$ up to third order in momentum. The expansion is

$$
\begin{aligned}
\phi(\boldsymbol{k}) & \simeq \frac{3 a}{2}\left(k_{y}-i k_{x}\right)+\frac{1}{2}\left(\frac{3 a}{2}\right)^{2}\left(k_{x}^{2}+k_{y}^{2} / 3+2 i k_{x} k_{y}\right) \\
& +\frac{1}{6}\left(\frac{3 a}{2}\right)^{3}\left(i k_{x}^{3}-k_{y}^{3} / 3-3 k_{x}^{2} k_{y}+i k_{y}^{2} k_{x}\right)
\end{aligned}
$$

The angular integral in Eq. (16) leads to

$$
\int_{0}^{2 \pi} d \theta\left([\Re \phi(\boldsymbol{k})]^{2}-[\Im \phi(\boldsymbol{k})]^{2}\right)=\frac{\pi}{24}\left(\frac{3 a k}{2}\right)^{4}
$$

where we still assume $|\phi(\boldsymbol{k})|=3 a k / 2$. Within this approximation the contribution to the conductivity coming from Eq. (16) has the form

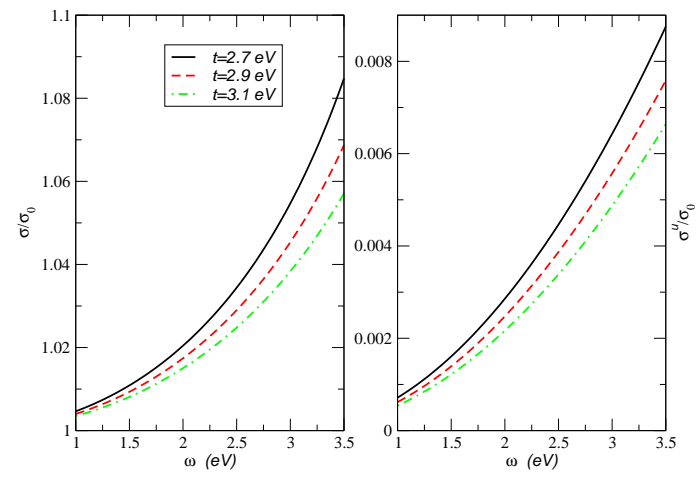

FIG. 3: (color online) Left: $\sigma(\omega) / \sigma_{0}$ as a function of the frequency, including both Eq. (19) and the correction $\Re \sigma_{x x}^{u}$, Eq. (28), for several values of $t$. Right: The correction $\Re \sigma_{x x}^{u}$, given by Eq. 28, for several values of $t$. It is clear that the contribution from this term has barely no effect on the results given by Eq. (19). The calculations are for zero chemical potential and for room temperature (there is no visible effect on $\sigma(\omega) / \sigma_{0}$ in the visible range of the spectrum, when compared to a zero temperature calculation).

\section{The imaginary part of the conductivity}

Neglecting the term proportional to Eq. (15), the imaginary part of the conductivity is given by

$$
\begin{aligned}
\Im \sigma_{x x}(\omega) & =\frac{1}{\hbar \omega} \frac{4}{\pi} \sigma_{0}\left(\mu-\frac{2}{9} \mu^{3} / t^{2}\right)-\frac{\sigma_{0}}{\pi} \log \frac{|\hbar \omega+2 \mu|}{|\hbar \omega-2 \mu|} \\
& -\frac{\sigma_{0}}{36 \pi}\left(\frac{\hbar \omega}{t}\right)^{2} \log \frac{|\hbar \omega+2 \mu|}{|\hbar \omega-2 \mu|}
\end{aligned}
$$

where we have included all the terms that diverge at $\hbar \omega=2 \mu$ and the contribution from the cubic term in frequency in the density of states. The contribution of the last term of $f[\phi(\boldsymbol{k})]$ in 13 is given by

$$
\Im \sigma_{x x}^{u}(\omega)=-\frac{\sigma_{0}}{18 \pi} \frac{1}{4 ! 2^{4}}\left(\frac{\hbar \omega}{t}\right)^{2} \log \frac{|\hbar \omega+2 \mu|}{|\hbar \omega-2 \mu|} .
$$

If we neglect the terms in $\mu^{3}$ and $\omega^{2}$ we obtain the same expressions as those derived by Falkovsky and Pershoguba 29 . We note that these terms are also obtained from the polarizability in the limit $q \rightarrow 0$ since the Fermi velocity is not $k$-dependent. ${ }^{12}$

\section{E. The Drude weight and the Hall coefficient}

$\Re \sigma_{x x}^{u}(\omega)=\sigma_{0} \frac{1}{4 ! 2^{4}}\left(\frac{\hbar \omega}{t}\right)^{2}\left(\tanh \frac{\hbar \omega+2 \mu}{4 k_{B} T}+\tanh \frac{\hbar \omega-2 \mu}{4 k_{B} T}\right)$

Due to the prefactor, this contribution has only a small effect and shows that the current operator basically conserves the circular symmetry found close to the $K$-points. In Figure 3 we present $\sigma(\omega) / \sigma_{0}$ as function of the frequency, considering several values of $t$, in the optical range and also discussing the numerical value of the term given in Eq. (28).
The Drude weight (or charge stiffness) defined by Eq. (9) can be computed in different limits. In the case $\mu=0$ we are interested in its temperature dependence. For zero temperature the exact relation

$$
\sum_{\boldsymbol{k}}|\phi(\boldsymbol{k})|=\frac{1}{8} \sum_{\boldsymbol{k}} \frac{f[\phi(\boldsymbol{k})]}{|\phi(\boldsymbol{k})|}
$$


assures that $D=0$ when $\mu=0$. In general, the Drude weight has the following form:

$$
\begin{aligned}
D(T, \mu) & =t \sigma_{0} \frac{4 \pi^{2}}{3 \sqrt{3}} \frac{1}{N_{c}} \sum_{\boldsymbol{k}}\left[|\phi(\boldsymbol{k})|-\frac{1}{8} \frac{f[\phi(\boldsymbol{k})]}{|\phi(\boldsymbol{k})|}\right] \\
& \times\left[\tanh \frac{t|\phi(\boldsymbol{k})|+\mu}{2 k_{B} T}+\tanh \frac{t|\phi(\boldsymbol{k})|-\mu}{2 k_{B} T}\right]
\end{aligned}
$$

In the case of finite $\mu$, the temperature dependence of $D(T, \mu)$ is negligible. In the Dirac cone approximation we obtain

$$
D(0, \mu)=4 \pi \sigma_{0} \mu\left[1-\frac{1}{9}\left(\frac{\mu}{t}\right)^{2}\right] .
$$

On the other hand, at zero chemical potential the temperature dependence of the charge stiffness is given by

$$
D(T, 0)=8 \pi \ln 2 \sigma_{0} k_{B} T-4 \pi \zeta(3) \sigma_{0} \frac{\left(k_{B} T\right)^{3}}{t^{2}},
$$

where $\zeta(x)$ is the Riemann zeta function.

Zotos et al. have shown a very general relation between the Drude weight and the Hall coefficient ${ }^{45,46}$ This relation is

$$
R_{H}=-\frac{1}{e D} \frac{\partial D}{\partial n} .
$$

Equation (35) does not take into account the possibility of valley degeneracy and therefore it has to be multiplied by two when we apply it to graphene. In the case of a finite chemical potential we have the following relations between the Fermi wave vector $k_{F}$ and the chemical potential: $n=k_{F}^{2} / \pi$ and $\mu=2 t a k_{F} / 3$. Applying Eq. (35) to graphene we obtain

$$
R_{H}=-\frac{2}{e} \frac{n^{-1 / 2} / 2-3 a^{2} \sqrt{n} / 8}{\sqrt{n}-a^{2} n^{3 / 2} / 4} \simeq-\frac{1}{e n} .
$$

\section{EFFECT OF $t^{\prime}$ ON THE CONDUCTIVITY OF GRAPHENE}

In this section we want to discuss the effect of $t^{\prime}$ on the conductivity of graphene. One important question is what the value of $t^{\prime}$ is in graphene. Deacon et al.$^{\frac{39}{}}$ proposed that the dispersion for graphene, obtained from a tight-binding approach with non-orthogonal basis functions, is of the form

$$
E= \pm \frac{t|\phi(\boldsymbol{k})|}{1 \mp s_{0}|\phi(\boldsymbol{k})|}
$$

with $|\phi(\boldsymbol{k})| \simeq \frac{3}{2} k a$, with $a$ the carbon-carbon distance. On the other hand the dispersion of graphene including $t^{\prime}$ has the form

$$
E= \pm t \frac{3}{2} k a-t^{\prime}\left[\frac{9}{4}(k a)^{2}-3\right] .
$$

To relate $t^{\prime}$ and $s_{0}$ we expand Eq. (37) as

$$
E \simeq \pm t|\phi(\boldsymbol{k})|\left(1 \pm s_{0}|\phi(\boldsymbol{k})|\right)= \pm t \frac{3}{2} k a+s_{0} t \frac{9}{4}(k a)^{2},
$$

which leads $t^{\prime} / t=-s_{0}$ with $s_{0}=0.13$.

For computing the conductivity of graphene we need to know the Green's function with $t^{\prime}$. These can be written in matrix form as

$$
\begin{aligned}
\mathbb{G}^{0}\left(\boldsymbol{k}, i \omega_{n}\right) & =\sum_{\alpha=+,-} \frac{1 / 2}{i \omega_{n}-\alpha t|\phi(\boldsymbol{k})| / \hbar+2 t^{\prime}\left[|\phi(\boldsymbol{k})|^{2}-3\right] / \hbar} \\
& \times\left(\begin{array}{cc}
1 & -\alpha \phi(\boldsymbol{k}) /|\phi(\boldsymbol{k})| \\
-\alpha \phi(\boldsymbol{k})^{*} /|\phi(\boldsymbol{k})| & 1
\end{array}\right), \quad(40)
\end{aligned}
$$

where $\mathbb{G}^{0}\left(\boldsymbol{k}, i \omega_{n}\right)$ stands for

$$
\mathbb{G}^{0}\left(\boldsymbol{k}, i \omega_{n}\right)=\left(\begin{array}{ll}
G_{A A}\left(\boldsymbol{k}, i \omega_{n}\right) & G_{A B}\left(\boldsymbol{k}, i \omega_{n}\right) \\
G_{B A}\left(\boldsymbol{k}, i \omega_{n}\right) & G_{B B}\left(\boldsymbol{k}, i \omega_{n}\right)
\end{array}\right) .
$$

From Eq. (40) we see that only the poles are modified, with the coherence factors having the same form as in the case with $t^{\prime}=0$. The current operator $j_{x}^{P}=j_{x, t}^{P}+j_{x, t^{\prime}}^{P}$, as derived from the tight-binding Hamiltonian is written in momentum space as

$$
\begin{aligned}
j_{x, t}^{P} & =\frac{t i e a}{2 \hbar} \sum_{\sigma} \sum_{\boldsymbol{k}}\left[(\phi(\boldsymbol{k})-3) a_{\sigma, \boldsymbol{k}}^{\dagger} b_{\sigma, \boldsymbol{k}}-\right. \\
& \left.-\left(\phi^{*}(\boldsymbol{k})-3\right) b_{\sigma, \boldsymbol{k}}^{\dagger} a_{\sigma, \boldsymbol{k}}\right],
\end{aligned}
$$

and

$$
\begin{aligned}
j_{x, t^{\prime}}^{P}= & \frac{3 t^{\prime} i e a}{2 \hbar} \sum_{\sigma} \sum_{\boldsymbol{k}}\left[\phi(\boldsymbol{k})-\phi^{*}(\boldsymbol{k})\right] \times \\
& \left(a_{\sigma, \boldsymbol{k}}^{\dagger} a_{\sigma, \boldsymbol{k}}+b_{\sigma, \boldsymbol{k}}^{\dagger} b_{\sigma, \boldsymbol{k}}\right) .
\end{aligned}
$$

The operators $j_{x, t}^{P}$ and $j_{x, t^{\prime}}^{P}$ are the current operators associated with the hopping amplitudes $t$ and $t^{\prime}$, respectively. The current-current correlation function is now a sum of three different terms: one where we have two $j_{x, t}^{P}$ operators, another one where we have a $j_{x, t}^{P}$ and a $j_{x, t^{\prime}}^{P}$, and a third one with two $j_{x, t^{\prime}}^{P}$. This last term vanishes exactly, since it would correspond to the current-current correlation function of a triangular lattice. Also the crossed term vanishes exactly which can be understood by performing a local gauge transformation to the fermionic operators of one sub-lattice, only. The first term leads to a contribution of the same form as in Eq. (19) but with the numerators of the two tanh replaced by $E_{+}=\hbar \omega+2 t^{\prime}\left[(\hbar \omega)^{2} /\left(4 t^{2}\right)-3\right]+2 \mu$ and $E_{-}=\hbar \omega-2 t^{\prime}\left[(\hbar \omega)^{2} /\left(4 t^{2}\right)-3\right]-2 \mu$, respectively.

As a consequence the effect of $t^{\prime}$ in the conductivity a graphene only enters in the band structure $E_{ \pm}$in the Fermi functions. In Figure 4 we plot the real part of the optical conductivity for two different values of $\mu$, one with the Fermi energy in the conduction band and the other with the Fermi energy in the valence band. There is a small effect near twice the absolute value of the chemical potential, due to the breaking of particle-hole symmetry introduced by $t^{\prime}$. For optical frequencies, the effect of $t^{\prime}$ is negligible. 


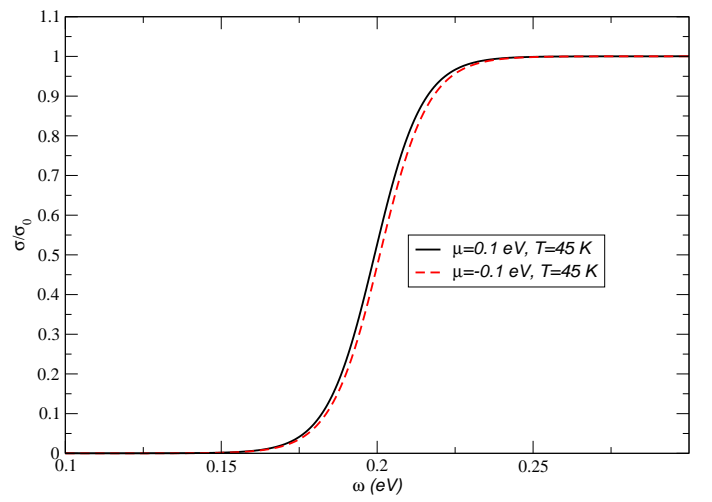

FIG. 4: (color online) Real part of the conductivity for two values of the chemical potential at the temperature of $45 \mathrm{~K}$. The parameters used are $t=3.1 \mathrm{eV}$ and $t^{\prime}=-0.13 t$. Only the energy range of $\omega \in$ $[0.1,0.3] \mathrm{eV}$ is shown because only here has the chemical potential difference any noticeable effect.

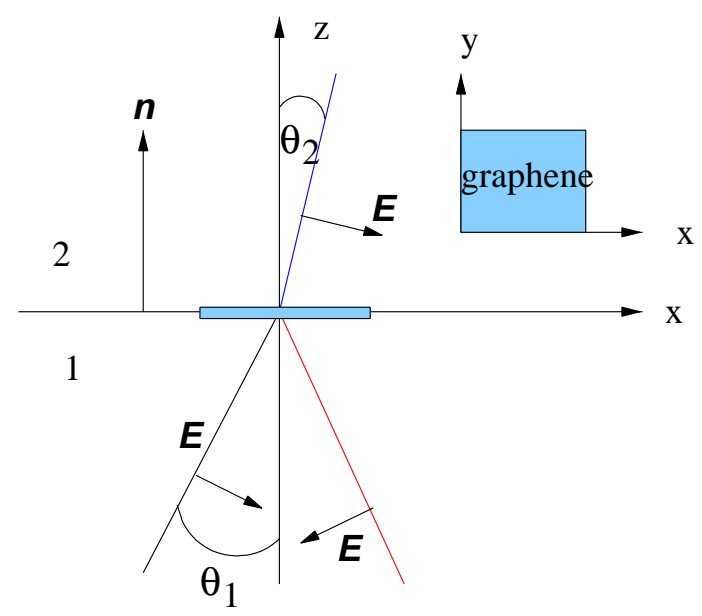

FIG. 5: (color online) Geometry of $p$ polarized light scattering between two media with graphene separating them. The electrical permittivities of the two media are $\epsilon_{i} \epsilon_{0}$, with $i=1,2$

\section{THE ELECTROMAGNETIC SCATTERING PROBLEM}

Here we derive the reflectivity and the transmissivity of light between two media, characterized by electrical permittivities $\epsilon_{i} \epsilon_{0}$, with $i=1,2$, separated by a graphene flake. The scattering geometry is represented in Fig. 5, i.e., we assume the field to propagate in the direction $\boldsymbol{k}=\left(k_{x}, 0, k_{z}\right)$.

In the following, we assume the field to be given by $\boldsymbol{E}=$ $\left(E_{x}, 0, E_{z}\right)$ ( $p$ polarization). The case of $s$ polarization is addressed in Appendix B.

The electromagnetic boundary conditions then are ${ }^{47}$

$$
\begin{aligned}
& \left(\boldsymbol{D}_{2}-\boldsymbol{D}_{1}\right) \cdot \boldsymbol{n}=\rho, \\
& \boldsymbol{n} \times\left(\boldsymbol{E}_{2}-\boldsymbol{E}_{1}\right)=0,
\end{aligned}
$$

where $\rho$ is the surface charge density, in our case the graphene charge density. If we represent the intensity of the incident, reflected, and transmitted electric field as $E_{i}, E_{r}$, and $E_{t}$, respectively, the boundary conditions can be written as

$$
\begin{array}{r}
\left(E_{i}-E_{r}\right) \cos \theta_{1}=E_{t} \cos \theta_{2}, \\
-\epsilon_{2} \epsilon_{0} E_{t} \sin \theta_{2}+\epsilon_{1} \epsilon_{0}\left(E_{i}+E_{r}\right) \sin \theta_{1}=\rho,
\end{array}
$$

where $\epsilon_{0}$ is the vacuum permittivity, $\epsilon_{1}$ and $\epsilon_{2}$ are the relative permittivity of the two media and $\theta_{1}$ and $\theta_{2}$ are the incident and refracted angle, respectively. Now the continuity equation in momentum space reads

$$
\rho(\omega)=j_{x}(\omega) k_{x} / \omega,
$$

and Ohm's law is written as

$$
j_{x}(\omega)=\sigma(\omega) E_{x}=\sigma(\omega) E_{t} \cos \theta_{2} .
$$

Combining Eqs. (46) - (49), we arrive at the following result, valid for normal incidence, for the transmissivity $T$

$$
T=\sqrt{\frac{\epsilon_{2}}{\epsilon_{1}}} \frac{4\left(\epsilon_{1} \epsilon_{0}\right)^{2}}{\left(\sqrt{\epsilon_{1} \epsilon_{2}}+\epsilon_{1}\right) \epsilon_{0}+\sqrt{\epsilon_{1}} \sigma(\omega) /\left.c\right|^{2}} .
$$

If we now consider both media to be vacuum and that the graphene is at half filling $\left(\sigma(\omega) \simeq \sigma_{0}\right)$ we obtain

$$
T=\frac{1}{(1+\pi \alpha / 2)^{2}} \simeq 1-\pi \alpha,
$$

where $\alpha=e^{2} /\left(4 \pi \epsilon_{0} c \hbar\right)$, is the fine structure constant. The reflectivity is also controlled by the fine structure constant $\alpha$. For normal incidence it reads

$$
R=\frac{\left|\sqrt{\epsilon_{1} \epsilon_{2}} \epsilon_{0}+\sqrt{\epsilon_{1}} \sigma(\omega) / c-\epsilon_{1} \epsilon_{0}\right|^{2}}{\left|\sqrt{\epsilon_{1} \epsilon_{2}} \epsilon_{0}+\sqrt{\epsilon_{1}} \sigma(\omega) / c+\epsilon_{1} \epsilon_{0}\right|^{2}},
$$

and if both media are the vacuum we obtain

$$
R=\frac{\pi^{2} \alpha^{2}}{4} T .
$$

In Fig. 6, the transmission and reflection coefficients for normal incident as function of the frequency for temperature $T=10 \mathrm{~K}$ are shown where the first medium is vacuum $\left(\epsilon_{1}=\right.$ $1)$ and the second medium is either vacuum $\left(\epsilon_{2}=1\right)$ or a $\mathrm{SiO}_{2}$-substrate $\left(\epsilon_{2}=\epsilon_{\infty}=2, \epsilon_{\infty}\right.$ being the high-frequency dielectric constant of $\mathrm{SiO}_{2}$ ). The left hand side shows the data for zero doping and the right hand side for finite doping $\mu=$ $0.2 \mathrm{eV}$. In Appendix B, we present the formulas for arbitrary angle of incidence.

It is interesting to compare the result for graphene with that for bilayer graphene. For the bilayer, the transmissivity is given by 19

$$
T=1-2 \pi \alpha f_{2}(\omega)
$$

with $f_{2}(\omega)$ given by

$$
\begin{aligned}
f_{2}(\omega) & =\frac{\hbar \omega+2 t_{\perp}}{2\left(\hbar \omega+t_{\perp}\right)}+\frac{\theta\left(\hbar \omega-t_{\perp}\right)}{\left(\hbar \omega / t_{\perp}\right)^{2}} \\
& +\frac{\left(\hbar \omega-2 t_{\perp}\right) \theta\left(\hbar \omega-2 t_{\perp}\right)}{2\left(\hbar \omega-t_{\perp}\right)},
\end{aligned}
$$



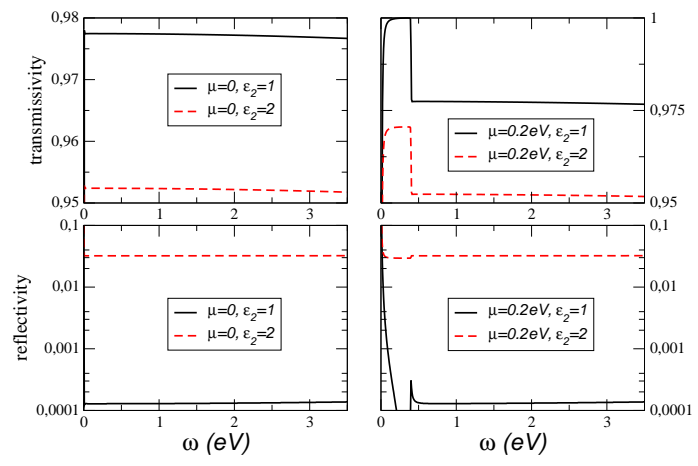

FIG. 6: (color online) The transmissivity and reflectivity for normal incident as function of the frequency for $T=10 \mathrm{~K}$ where the first medium is vacuum $\left(\epsilon_{1}=1\right)$ and the second medium is either vacuum $\left(\epsilon_{2}=1\right)$ or a $\mathrm{SiO}_{2}$-substrate $\left(\epsilon_{2}=\epsilon_{\infty}=2\right)$. Left: At zero chemical potential. Right: At finite chemical potential $\mu=0.2 \mathrm{eV}$.

and $t_{\perp}$ the hopping amplitude between the graphene planes. For frequencies much larger than $t_{\perp}$, which is the case in an experiment done in the visible region of the spectrum, one obtains

$$
f_{2}(\omega) \simeq 1-\frac{t_{\perp}^{2}}{(\hbar \omega)^{2}} \simeq 1
$$

which leads to $T \simeq 1-2 \pi \alpha$. Again, as in graphene, the transmissivity is controlled by the fine structure constant. It is interesting to note that for $\hbar \omega \ll t_{\perp}$ we also obtain the same result for $T$.

The appearance of the fine structure constant $\alpha$ in the two cases is connected to the spinorial structure of the electronic wave function. In other words, the reduction of the transmissivity through a clean system is caused by a universal current induced by interband transitions.

\section{CONCLUSIONS}

We have presented a detailed study of the optical properties of graphene based on the general, non-interacting tightbinding model. Special emphasis was placed on going beyond the usual Dirac-cone approximation, i.e., we included the cubic term in the density-of-states. The conductivity was thus consistently calculated to order $(\hbar \omega / t)^{2}$ for arbitrary chemical potential and temperature.

We also assessed the effect of the next nearest neighbor coupling $t^{\prime}$ on the optical properties. We find that the additional terms to the current operator do not contribute to the conductivity and that modifications only enter through the modified energy dispersion.

Using the full conductivity of clean graphene, we determine the transmissivity and reflectivity of light that is scattered from two media with different permittivity and graphene at the interface. Our results are important for optical experiments in the visible frequency range..$^{44}$ For example, the apparent dis- agreement between the presented theory for graphene and experiments by Dawlaty et al..$^{42}$ at visible frequencies indicates that the interlayer interaction in epitaxial-SiC graphene is significant and cannot be neglected.

\section{Acknowledgements}

This work was supported by the ESF Science Programme INSTANS 2005-2010, and by FCT under the grant PTDC/FIS/64404/2006.

\section{APPENDIX A: EQ. 16 UP TO FIRST ORDER IN MOMENTUM}

The function $\phi(\boldsymbol{k})$ is given close to the Dirac point by

$$
\phi(\boldsymbol{k}) \simeq \frac{3 a}{2}\left(k_{y}-i k_{x}\right) .
$$

This leads to the following result

$$
T(\theta)=\frac{[\Re \phi(\boldsymbol{k})]^{2}-[\Im \phi(\boldsymbol{k})]^{2}}{|\phi(\boldsymbol{k})|^{2}}=-\cos (2 \theta)
$$

It is now easy to see that

$$
\int_{0}^{2 \pi} d \theta T(\theta) g(|\phi(\boldsymbol{k})|)=0
$$

where we have used the result $|\phi(\boldsymbol{k})|=3 a k / 2$, valid near the Dirac point.

\section{APPENDIX B: TRANSMISSIVITY AND REFLECTIVITY FOR ARBITRARY INCIDENCE}

Here we present the general formula for the transmissivity and reflectivity of light being scattered at a plane surface between two media of different dielectric properties and a graphene sheet at the interface.

For $p$ polarization, the reflection and transmission amplitude are obtained from the boundary conditions of Eqs. 46, (47) and read

$$
r=\frac{M-1}{M+1}, t=\sqrt{\frac{\epsilon_{1}}{\epsilon_{2}}} \frac{2 K}{M+1}
$$

with $M=K+\Sigma \cos \theta_{1}$, where $\theta_{1}$ denotes the incident angle and

$$
K=\frac{\epsilon_{2}}{\epsilon_{1}} \frac{k_{z}^{i}}{k_{z}^{t}}, \Sigma=\frac{\sigma(\omega)}{\sqrt{\epsilon_{1}} \epsilon_{0} c} .
$$

Above, $k_{z}^{i}=\sqrt{\epsilon_{1}(\omega / c)^{2}-k_{x}^{2}}\left(k_{z}^{t}=\sqrt{\epsilon_{2}(\omega / c)^{2}-k_{x}^{2}}\right)$ denotes the perpendicular component of the incident (transmitted) wave vector relative to the interface, $k_{x}$ the parallel (conserved) component and $\epsilon_{1}\left(\epsilon_{2}\right)$ is the dielectric constant of the first (second) medium, see Fig. 5. For $s$ polarization, $r$ and $t$ 
are independent of the angle of incident and in the Dirac cone approximation yield the same result as for $p$ polarization in the case of normal incident $\left(\theta_{1}=0\right)$.

Generally, the reflection and the transmission coefficient are given by $R=|r|^{2}$ and $T=|t|^{2} k_{z}^{t} / k_{z}^{i}$, respectively. For a simple (non-conducting) interface, this leads to the conservation law $T+R=1$. Notice that there is no such conservation in the present case due to absorption within the graphene sheet.
For a suspended graphene sheet with $\epsilon_{1}=\epsilon_{2}=1$ at the Dirac point $\left(\sigma(\omega) \simeq \sigma_{0}\right)$, the reflection and transmission coefficient for $p$ polarization read

$$
R=\frac{\left(\tilde{\alpha} \cos \theta_{1}\right)^{2}}{\left(1+\tilde{\alpha} \cos \theta_{1}\right)^{2}}, T=\frac{1}{\left(1+\tilde{\alpha} \cos \theta_{1}\right)^{2}},
$$

with $\tilde{\alpha}=\pi \alpha / 2$ and $\alpha=e^{2} /\left(4 \pi \epsilon_{0} c \hbar\right)$ the fine structure constant.
${ }^{1}$ K. S. Novoselov, A. K. Geim, S. V. Morozov, D. Jiang, Y. Zhang, S. V. Dubonos, I. V. Grigorieva, and A. A. Firsov, Science 306, 666 (2004).

${ }^{2}$ K. S. Novoselov, D. Jiang, T. Booth, V.V. Khotkevich, S. M. Morozov, A. K. Geim, PNAS 102, 10451 (2005).

3 A. K. Geim and K. S. Novoselov, Nature Materials 6, 183 (2007).

4 A. H. Castro Neto, F. Guinea, and N. M. R. Peres, Phys. World 19, 33 (2006).

5 A. H. Castro Neto, F. Guinea, N. M. R. Peres, K. S. Novoselov, and A. K. Geim, arXiv:0709.1163

${ }^{6}$ C. W. Beenakker, arXiv:0710.3848

7 V. P. Gusynin, S. G. Sharapov, Phys. Rev. B 73, 245411 (2006).

8 V. P. Gusynin, S. G. Sharapov, and J. P. Carbotte, Phys.Rev.Lett. 96, 256802 (2006).

9 V. P. Gusynin, S. G. Sharapov, and J. P. Carbotte, Phys.Rev.Lett. 98, 157402 (2007).

10 V. P. Gusynin, S. G. Sharapov, and J. P. Carbotte, Phys. Rev. B 75, 165407 (2007).

11 V. P. Gusynin, S. G. Sharapov, and J. P. Carbotte, Int. Jour. of Mod. Phys. B 21, 4611 (2007).

12 B. Wunsch, T. Stauber, F. Sols, and F. Guinea, New J. Phys. 8, 318 (2006).

13 E. H. Hwang and S. Das Sarma, Phys. Rev. B 75, 205418 (2007).

14 N. M. R. Peres, F. Guinea, and A. H. Castro Neto, Phys. Rev. B 73, 125411 (2006).

15 I. F. Herbut, V. Juricic, and O. Vafek, Phys. Rev. Lett. 100, 046403 (2008).

${ }^{16}$ L. Fritz, J. Schmalian, M. Mueller, and S. Sachdev, Phys. Rev. B 78, 085416 (2008).

17 Mikito Koshino and Tsuneya Ando, Phys. Rev. B 73, 245403 (2006).

18 Johan Nilsson, A. H. Castro Neto, F. Guinea, and N. M. R. Peres, Phys. Rev. Lett. 97, 266801 (2006); Phys. Rev. B 78, 045405 (2008).

19 D. S. L. Abergel and Vladimir I. Fal'ko, Phys. Rev. B 75, 155430 (2007).

20 T. Stauber, N. M. R. Peres, F. Guinea, and A. H. Castro Neto, Phys. Rev. B 75, 115425 (2007).

${ }^{21}$ Eduardo V. Castro, K. S. Novoselov, S. V. Morozov, N. M. R. Peres, J.M.B. Lopes dos Santos, Johan Nilsson, F. Guinea, A. K. Geim, and A. H. Castro Neto, Phys. Rev. Lett. 99, 216802 (2007).

22 Jeroen B. Oostinga, Hubert B. Heersche, Xinglan Liu, Alberto F. Morpurgo, and Lieven M. K. Vandersypen, Nature Materials 7, 151 (2007).

${ }^{23}$ E. J. Nicol and J. P. Carbotte, Phys. Rev. B 77, 155409 (2008).

${ }^{24}$ N. M. R. Peres, J. M. B. Lopes dos Santos, and T. Stauber, Phys. Rev. B 76, 073412 (2007).
25 T. Stauber, N. M. R. Peres, and F. Guinea, Phys. Rev. B 76, 205423 (2007).

${ }^{26}$ F. T. Vasko and V. Ryzhii, Phys. Rev. B 76, 233404 (2007).

27 F. T. Vasko and V. Ryzhii, Phys. Rev. B 77, 195433 (2008).

${ }^{28}$ L. A. Falkovsky and A. A. Varlamov, Eur. Phys. J. B 56, 281 (2007).

${ }^{29}$ L. A. Falkovsky and S. S. Pershoguba, Phys. Rev. B 76, 153410 (2007).

30 L. A. Falkovsky, arXiv:0806.3663, to be published in "DubnaNano2008".

31 A. W. W. Ludwig, M. P. A. Fisher, R. Shankar, and G. Grinstein, Phys. Rev. B 50, 7526 (1994).

32 N. M. R. Peres and T. Stauber, Int. J. Mod. Phys. B 22, 2529 (2008).

33 T. Stauber and N. M. R. Peres, J. Phys.: Condens. Matter 20, 055002 (2008).

${ }^{34}$ Nguyen Hong Shon and Tsuneya Ando, J. Phys. Soc. Jpn. 67, 2421 (1998).

35 Tsuneya Ando, Yisong Zheng, and Hidekatsu Suzuura J. Phys. Soc. Jpn. 71,1318 (2002).

36 A. B. Kuzmenko, E. van Heumen, F. Carbone, and D. van der Marel, Phys. Rev. Lett. 100, 117401 (2008).

37 Thomas G. Pedersen, Phys. Rev. B 67, 113106 (2003).

38 Z. Jiang, E. A. Henriksen, L. C. Tung, Y.-J. Wang, M. E. Schwartz, M. Y. Han, P. Kim, and H. L. Stormer, Phys. Rev. Lett. 98, 197403 (2007).

39 R. S. Deacon, K-C. Chuang, R. J. Nicholas, K. S. Novoselov, and A. K. Geim, Phys. Rev. B 76, 081406(R) (2007).

40 Z. Q. Li, E. A. Henriksen, Z. Jiang, Z. Hao, M. C. Martin, P. Kim, H. L. Stormer, and D. N. Basov, Nature Physics 4, 532 (2008).

${ }^{41}$ T. Stauber, N. M. R. Peres, and A. H. Castro Neto, Phys. Rev. B 78, 085418 (2008).

42 Jahan M. Dawlaty, Shriram Shivaraman, Jared Strait, Paul George, Mvs Chandrashekhar, Farhan Rana, Michael G. Spencer, Dmitry Veksler, and Yunqing Chen, arXiv:0801.3302.

43 P. Plochocka, C. Faugeras, M. Orlita, M. L. Sadowski, G. Martinez, M. Potemski, M. O. Goerbig, J.-N. Fuchs, C. Berger, W. A. de Heer, Phys. Rev. Lett. 100, 087401 (2008).

${ }^{44}$ R. R. Nair, P. Blake, A. N. Grigorenko, K. S. Novoselov, T.J. Booth, T. Stauber, N. M. R. Peres, and A. K. Geim, Science 320, 1308 (2008).

45 X. Zotos, F. Naef, M. Long, and P. Prelovsek, Phys. Rev. Lett. 85, 377 (2000).

46 P. Prelovsek and X. Zotos, Phys. Rev. B 64, 235114 (2001).

47 John David Jackson, Classical Electrodynamics, (Wiley, 2001, $3^{r d}$ edition), p. 16 . 